\title{
Prognosis of patients with postural tachycardia syndrome: a follow-up study
}

\author{
Sa-Yoon Kang, Hong Jun Kim, and Keun Hyuk Ko \\ Department of Neurology, Jeju National University School of Medicine, Jeju, Korea
}

Received: June 26, 2018

Revised: December 20, 2018

Accepted: December 25, 2018

\section{Correspondence to}

\section{Sa-Yoon Kang}

Department of Neurology, Jeju National University School of Medicine, 102 Jejudaehak-ro, Jeju 63243, Korea

Tel: +82-64-754-8175

Fax: +82-64-717-1630

E-mail: neurokang@jejunu.ac.kr

\section{ORCID}

\section{Sa-Yoon Kang}

http://orcid.org/0000-0001-6755-089X

\section{Hong Jun Kim}

http://orcid.org/0000-0003-2623-5951

\section{Keun Hyuk Ko}

http://orcid.org/0000-0001-6453-3461

\section{INTRODUCTION}

Postural tachycardia syndrome (POTS) is defined by the presence of orthostatic intolerance symptoms associated with a sustained heart rate increase of more than 30 beats/min on head-up tilt (HUT), without orthostatic hypotension. ${ }^{1,2}$ Symptoms related to orthostatic changes usually include light-headedness, palpitations and tremor, blurred vision, head- 
ache, and neck pain, as well as syncope. POTS occurs most commonly in young women of child-bearing age and the clinical presentation is heterogeneous and non-specific, probably reflecting multiple underlying mechanisms. ${ }^{3-5}$

A detailed clinical evaluation should be carried out prior to HUT testing to exclude other conditions including excessive venous pooling, hypovolemia, and deconditioning, which may cause orthostatic intolerance. It is important to recognize POTS earlier as proper treatment significantly improves autonomic and hemodynamic disturbances, but long-term prognosis remains uncertain. ${ }^{6}$ The main aim of the current study was to determine how autonomic parameters and clinical features can change from baseline to follow-up in patients with POTS.

\section{MATERIALS AND METHODS}

We enrolled patients who met all of the following criteria: 1) symptoms of orthostatic intolerance, including light-headedness, palpitations, blurred vision, tremor, and difficulty in concentration, 2) sustained increase in the heart rate of 30 beats/min or increase of heart rate to $\geq 120$ beats/min within the first 10 minutes of HUT and without significant orthostatic hypotension. For individuals who were younger than 19 years of age, the required heart rate increment was at least 40 beats/min. The exclusion criteria were as follows: the presence of systemic illness, the presence of known causes of autonomic failure, pregnant women, and taking anticholinergic agents, adrenergic antagonists, or other medications that could not be suspended before testing. We collected the data such as the duration of symptoms, precipitating event, and treatment status from the medical records of each patients. All patients provided their written informed consent prior to participation in the study, which was approved by the Ethics Committee of Jeju National University Hospital.

Standardized autonomic function tests were performed. All included patients were assessed using a Q-Sweat HRV (WR Medical Electronics Co., Maplewood, MN, USA) and Finometer (Finapres Medical System, Amsterdam, Netherland). For at least 12 hours prior to the study, all patients discontinued any drugs that might influence the autonomic function tests. The changes in the heart rate in response to deep breathing and the Valsalva ratio were used to assess cardiovagal function. The cardiovascular adrenergic function was estimated by measuring the blood pressure and heart rate responses to the Valsalva maneuver and HUT. The quantitative sudomotor axon reflex test (QSART) was used to evaluate the postganglionic sympathetic sudomotor axon function. First, QSART was performed with Q-Sweat. lontophoresed acetylcholine was infused into the skin to stimulate the postganglionic sudomotor unmyelinated nerves. The produced sweat was collected and quantified in a sudorometer. Four sites were measured in all patients: forearm (3/4 of the distance from the ulnar epicondyle to the pisiform bone); proximal leg ( $5 \mathrm{~cm}$ distal to the fibular head on the lateral side); distal leg $(5 \mathrm{~cm}$ proximal to the medial malleolus on the medial side); and foot (over the extensor digitorum brevis muscle). Subsequently, a deep breathing test was conducted as follows: the patient sat quietly and was instructed to breath smoothly, slowly, and deeply at 6 breaths/min (5 seconds inspiration and 5 seconds expiration), a rate which produces maximum variation of heart rate. The 10-second long respiratory cycle was repeated eight times. The E:I ratio is the ratio of the longest $\mathrm{R}$ - $\mathrm{R}$ interval during expiration to the shortest R-R interval during inspiration. Next, we performed the Valsalva maneuver. The intrathoracic pressure was kept at $40 \mathrm{mmHg}$ for 15 seconds by blowing through a mouthpiece connected to a mercury manometer. The changes in systolic blood pressure and R-R intervals were divided into four phases. The Valsalva ratio was calculated as the maximum $R-R$ interval in phase IV divided by the minimum $R-R$ interval in phase II. Lastly, we conducted the HUT test. After resting in a supine position for at least 5 minutes, subjects were tilted up to $70^{\circ}$ to assess the changes in systolic blood pressure at $70^{\circ}$ compared to the baseline level in the supine position. Cardiovascular recordings were collected using a noninvasive monitoring device during the head-up tilt test.

The composite autonomic symptom score 31 (COMPASS-31) reflects the overall severity of autonomic symptoms and includes 6 subscale scores to assess the severity of symptoms. COMPASS-31 quantifies 6 domains: orthostatic intolerance, vasomotor, secremotor, gastrointestinal, bladder, and pupillomotor domains. The 6 subscales add up to a total of 100, with COMPASS-31 score between 0 and 100, and high values represent severe symptoms. Each score is normalized for the confounding variables of age and sex. 
The Composite Autonomic Severity Score (CASS) is evaluated from the autonomic function tests and CASS is a measure of the severity and distribution of autonomic failure. Patients with a CASS of 3 or less have only mild autonomic failure. We compared the change in CASS and COMPASS-31 scores between the baseline and follow-up evaluations.

Paired data were analyzed using the Wilcoxon signed rank test to determine significant differences between two continuous variables. The chi-square test and Spearman test were used to determine significant correlation between the variables. All statistical analyses were conducted using IBM SPSS ver. 18.0 (IBM Corp., Armonk, NY, USA). Values are presented as the mean \pm standard deviation and the level of significance was defined as a $p<0.05$.

\section{RESULTS}

Sixty-eight patients who met the criteria for POTS were enrolled and 45 patients completed the follow-up evaluation by at least 1 year following the initial diagnosis. Among the 68 patients, 57 patients (84\%) were women. Most patients were young ( $21.2 \pm 9.8$ years of age). The mean duration of symptoms before the initial diagnosis was 13.1 months and three patients reported an antecedent viral infection before onset of symptoms. A precipitating event was present in 12 patients. The most common precipitating factor was exercise and the others were upper respiratory infection, emotional stress, heat exposure, and overeating. The main clinical characteristics are presented in Table 1. Most patients underwent conservative measures to treat their orthostatic symptoms at baseline assessment. The conservative measures included increased fluid and salt intake, compression garments, and physical counter-maneuvers. Some patients were taking beta-blockers (five patients) and pyridostigmine (three patients) at baseline and follow-up. The dosages were not significantly different at follow-up compared with the baseline. At follow-up, The mean duration of follow-up was $16 \pm 3$ months.

Comparing the changes in COMPASS-31 score between the baseline (30.2 \pm 23.2$)$ and follow-up (21.4 \pm 17.1$)$, a clinically significant improvement was seen in orthostatic symptoms during the follow-up period. Twelve patients were completely asymptomatic, 30 patients underwent im- provement, and only three patients remained highly symptomatic (Table 2). The increase in heart rate during HUT did not show significant difference between the baseline (36.4 \pm 13.6 beats $/ \mathrm{min})$ and follow-up $(33.2 \pm 14.1)$. Interestingly,

Table 1. Clinical characteristics of patients with POTS

\begin{tabular}{lcc}
\hline Feature & Finding & Patient \\
\hline Age (years) & $21.2 \pm 9.8$ & $68(100)$ \\
Sex & & \\
Female/male & & \\
\hline Symptom duration (months) & $13.1 \pm 10.7$ & $68(100)$ \\
Symptom onset & & \\
Acute (<1 month) & $23(33)$ \\
Subacute (1-3 months) & $18(26)$ \\
Insidious (>3 months) & $27(41)$ \\
Preceding events & \\
Upper respiratory infection & & $3(4)$ \\
Exercise & & $5(8)$ \\
Emotional stress & $3(4)$ \\
Heat exposure & $2(3)$ \\
Overeating & $2(3)$ \\
Unknown & & $53(78)$ \\
Body mass index (kg/m ${ }^{2}$ ) & 68 \\
$<18.5$ & & 10 \\
18.5-24.9 & & 49 \\
$25-29.9$ & $20.8 \pm 6.3$ & 6 \\
$30-34.9$ & 3 \\
\hline
\end{tabular}

Values are presented as mean \pm standard deviation or number (\%) unless otherwise indicated.

Table 2. COMPASS-31 scores at baseline and follow-up

\begin{tabular}{lcccc}
\hline \multirow{2}{*}{$\begin{array}{c}\text { COMPASS-31 } \\
\text { symptoms }\end{array}$} & $\begin{array}{c}\text { Baseline COM- } \\
\text { PASS-31 }\end{array}$ & & $\begin{array}{c}\text { Follow-up COM- } \\
\text { PASS-31 }\end{array}$ & p-value \\
\cline { 2 - 2 } $\begin{array}{c}\text { Score } \\
\text { Orthostatic } \\
\text { intolerance }\end{array}$ & $12.6 \pm 11.8$ & & $6.7 \pm 5.8$ & 0.01 \\
Vasomotor & $1.6 \pm 2.1$ & & $1.5 \pm 1.3$ & 0.54 \\
Secremotor & $5.4 \pm 6.9$ & & $5.4 \pm 3.5$ & 0.99 \\
Gastrointestinal & $6.6 \pm 5.4$ & & $5.0 \pm 3.9$ & 0.39 \\
Bladder & $1.1 \pm 1.6$ & & $1.0 \pm 1.8$ & 0.86 \\
Pupillomotor & $1.7 \pm 1.4$ & & $1.5 \pm 1.1$ & 0.71 \\
\hline Total score & $30.2 \pm 23.2$ & & $21.4 \pm 17.1$ & 0.04 \\
\hline
\end{tabular}

Values are presented as mean \pm standard deviation. COMPASS, composite autonomic symptom score. 
14 patients did not demonstrate a heart rate increase compatible with the criteria for POTS on follow-up HUT testing. Autonomic dysfunction was mild at both baseline and follow-up with a CASS of 2 or lower in 63 patients (94\%) and 43 patients (96\%), respectively. We found most patients had a mild autonomic impairment characterized by post-ganglionic sudomotor denervation in the lower limbs and adrenergic impairment. Cardiovagal parasympathetic dysfunction was minimal at baseline and follow-up (Table 3).

\section{DISCUSSION}

POTS is a condition characterized by abnormal persistent orthostatic tachycardia. The pathophysiological basis is heterogeneous in nature, explaining its myriad manifestations. ${ }^{8-10}$

Table 3. Autonomic dysfunction on clinical autonomic testing at baseline and follow-up

\begin{tabular}{|c|c|c|c|}
\hline \multirow[b]{2}{*}{ Test score } & \multicolumn{2}{|c|}{ Patients } & \multirow[b]{2}{*}{$p$-value } \\
\hline & $\begin{array}{c}\text { Baseline } \\
(n=68)\end{array}$ & $\begin{array}{l}\text { Follow-up } \\
(n=45)\end{array}$ & \\
\hline CASS (cardiovagal) & & & 0.97 \\
\hline 0 & $61(91)$ & $41(92)$ & \\
\hline 1 & $4(5)$ & $2(4)$ & \\
\hline$\geq 2$ & $3(4)$ & $2(4)$ & \\
\hline CASS (adrenergic) & & & 0.94 \\
\hline 0 & $56(82)$ & $36(80)$ & \\
\hline 1 & $8(11)$ & $6(13)$ & \\
\hline$\geq 2$ & $4(7)$ & $3(7)$ & \\
\hline CASS (sudomotor) & & & 0.89 \\
\hline 0 & $46(67)$ & $31(69)$ & \\
\hline 1 & $15(22)$ & $9(20)$ & \\
\hline$\geq 2$ & $7(11)$ & $5(11)$ & \\
\hline CASS (total) & & & 0.24 \\
\hline 0 & $42(62)$ & $30(68)$ & \\
\hline 1 & $8(12)$ & $7(15)$ & \\
\hline 2 & $10(15)$ & $5(11)$ & \\
\hline 3 & $5(8)$ & $2(4)$ & \\
\hline 4 & $2(2)$ & $1(2)$ & \\
\hline 6 & $1(1)$ & $0(0)$ & \\
\hline
\end{tabular}

Values are presented as number (\%).

CASS, composite autonomic severity score.
Overlapping conditions such as vasovagal syncope should be considered during the initial evaluation. Since POTS significantly impacts the quality of health, it is important to recognize this condition earlier. Some useful treatment options exist, but many suggested treatments have poor evidence base. $^{2,11}$ The prognosis of patients with POTS is favorable according to a prior study, which found that $80 \%$ of patients improved and $60 \%$ had minimal residual symptoms over a duration of approximately five years. ${ }^{6}$ However, there are few studies that have investigated the prognosis of patients with POTS prospectively.

Our study demonstrated that patients presenting with POTS can improve substantially and 14 patients no longer met the orthostatic heart rate criteria for POTS. Therefore, markedly delayed HUT at the initial diagnosis may have reduced the specificity of the POTS diagnosis despite orthostatic intolerance symptoms. Other major findings of our study suggested that the autonomic dysfunction in POTS patients was mild at baseline and during the follow-up period. The most common symptoms in our patients were dizziness, blurred vision, and syncope. Our study showed female predominance, with a female-male ratio of approximately 5:1 and a mean age of 21 years. The spectrum of symptoms and clinical characteristics of the patients were similar to previously published data with respect to age and sex. ${ }^{3,12,13}$ Contrary to previous studies, 3,6 a precipitating event was present only in a quarter of patients, probably due to delayed evaluation of orthostatic intolerance symptoms.

The improvement of orthostatic symptoms over time is consistent with prior studies. ${ }^{6,14}$ Though most patients reported overall improvement in the symptoms, the orthostatic heart rate remained elevated on follow-up HUT. The exact reason regarding why orthostatic symptoms had improved in these patients despite maintaining the heart rate increment is not certain. It is presumed that several factors could affect this finding, such as life-style modifications, applications of counter-maneuvers or medications. On follow-up, 14 patients (31\%) had a heart rate increment of less than 30 beats/min. Among these patients, three patients were being treated with beta-blockers, but the other patients had non-pharmacologic treatment. Our patients initially underwent non-pharmacologic therapy, which included education and reassurance, and application of counter-maneuvers. Almost all patients continued non-pharmacologic treatment 
and only eight patients remained on drug treatment at follow-up. More than a third of patients were completely asymptomatic and more than half reported a significant improvement, with only three patients remaining highly symptomatic. Accordingly, therapy based on autonomic principles, such as volume expansion, and physical counter-maneuvers, would seem reasonable.

The severity of autonomic dysfunction in our patients was mild, with only eight patients who had a CASS of 3 or higher at baseline. At follow-up, only three patients had a CASS that was higher than 3. The degree of other autonomic dysfunction assessed by autonomic function testing was mild, and was commonly accompanied by post-ganglionic sudomotor denervation. These results are in accordance with previous studies, which reported similar findings. ${ }^{3,14}$ Contrary to previous studies, our study included younger patients and showed a difference in preceding events. A few patients experienced infectious illness prior to symptom onset in our study. Our study supports that some POTS patients have limited autonomic neuropathy.

This study has several limitations. First of all, we included a relatively small number of patients. Most patients diagnosed with POTS had mild autonomic symptoms and did not want to participate in our study. In addition, we did not measure supine and standing norepinephrine levels. Although there has been some disagreement in the literature, there was a positive correlation between the heart rate increment on HUT testing and standing norepinephrine levels.

In conclusion, POTS presents as a heterogeneous clinical entity with a generally benign outcome. In most patients in our study, the orthostatic symptoms had substantially improved, although the mean orthostatic heart rate increment remained high on HUT. The autonomic dysfunction in our patients was mild and showed evidence of sympathetic denervation in the lower limbs, characterized by a limited autonomic neuropathy. These findings did not change during the follow-up period. Our study demonstrates that patients with POTS improve in orthostatic intolerance and show a favorable prognosis.

\section{Acknowledgements}

This work was supported by a research grant from Jeju National University Hospital in 2015.

\section{Conflicts of Interest}

The authors have no conflicts to disclose.

\section{REFERENCES}

1. Low PA, Opfer-Gehrking TL, Textor SC, Benarroch EE, Shen WK, Schondorf R, et al. Postural tachycardia syndrome (POTS). Neurology 1995;45(4 Suppl 5):S19-S25.

2. Carew S, Connor MO, Cooke J, Conway R, Sheehy C, Costelloe A, et al. A review of postural orthostatic tachycardia syndrome. Europace 2009;11:18-25.

3. Thieben MJ, Sandroni P, Sletten DM, Benrud-Larson LM, Fealey $\mathrm{RD}$, Vernino $\mathrm{S}$, et al. Postural orthostatic tachycardia syndrome: the Mayo clinic experience. Mayo Clin Proc 2007;82:308-313.

4. Stewart JM. Chronic orthostatic intolerance and the postural tachycardia syndrome (POTS). J Pediatr 2004;145:725-730.

5. Grubb BP, Kanjwal Y, Kosinski DJ. The postural tachycardia syndrome: a concise guide to diagnosis and management. J Cardiovasc Electrophysiol 2006;17:108-112.

6. Sandroni P, Opfer-Gehrking TL, McPhee BR, Low PA. Postural tachycardia syndrome: clinical features and follow-up study. Mayo Clin Proc 1999;74:1106-1110.

7. Sletten DM, Suarez GA, Low PA, Mandrekar J, Singer W. COMPASS 31: a refined and abbreviated composite autonomic symptom score. Mayo Clin Proc 2012;87:1196-1201.

8. Jacob G, Costa F, Shannon JR, Robertson RM, Wathen M, Stein M, et al. The neuropathic postural tachycardia syndrome. N Engl J Med 2000;343:1008-1014.

9. Furlan R, Jacob G, Snell M, Robertson D, Porta A, Harris P, et al. Chronic orthostatic intolerance: a disorder with discordant cardiac and vascular sympathetic control. Circulation 1998;98:154159.

10. Raj SR, Biaggioni I, Yamhure PC, Black BK, Paranjape SY, Byrne DW, et al. Renin-aldosterone paradox and perturbed blood volume regulation underlying postural tachycardia syndrome. Circulation 2005;111:1574-1582.

11. Freitas J, Santos R, Azevedo E, Costa O, Carvalho M, de Freitas AF. Clinical improvement in patients with orthostatic intolerance after treatment with bisoprolol and fludrocortisone. Clin Auton Res 2000;10:293-299.

12. Low PA, Sandroni P, Joyner MJ, Shen WK. Postural tachycardia syndrome (POTS). J Cardiovasc Electrophysiol 2009;20:352-358.

13. Sousa A, Lebreiro A, Freitas J, Maciel MJ. Long-term follow-up 
of patients with postural tachycardia syndrome. Clin Auton Res 2012;22:151-153

14. Kimpinski K, Fiqueroa JJ, Singer W, Sletten DM, lodice V, Sandroni P,
NEUROPHYSIOLOGY

et al. A prospective, 1-year follow-up study of postural tachycardia syndrome. Mayo Clin Proc 2012;87:746-752. 